\title{
An epidemiological study of the relationship between diet in the past and bone mineral density based on a survey of women aged 50 years and over in two public health centers, Ibaraki Prefecture
}

\author{
Kazuo MOTEGI', Satoshi TOYOKAW ${ }^{2}$, Hiroaki NISHIKAWA ${ }^{3}$, \\ Kazuko $\mathrm{OHKI}^{2}$, and Katsumi $\mathrm{KANO}^{4}$
}

The purpose of this study was to examine the relationship between bone mineral density (BMD) and past dietary habits of middle-aged and elderly women who were high-risk population of osteoporosis. We examined dietary habits of 713 women aged 50 years and over who underwent a check-up for osteoporosis at two public health centers in Ibaraki Prefecture. Items examined were frequency of intake of milk, cheese, yogurt, small fish, eggs, cooked beans, tofu, natto, vegetables and seaweed in their 20's, 30's and 40's. $\Sigma$ GS/D values were used as the indicator of BMD. Women with $\Sigma \mathrm{GS} / \mathrm{D}$ values less than 2.3 were designated as the low BMD group, and those with $\Sigma$ GS/D values more than 2.3 were designated as the control group. The differences in the intake frequencies of the aforementioned foods by the two groups were evaluated by chi-square tests. The results of our study showed that each proportion of people who consumed milk, small fish or seaweed every day in their 30's and 40's, and also in their 20's for the case of milk, was higher in the control group significantly. It was also indicated that each proportion of people who consumed tofu or vegetable more than three times in a week was higher in the control group. Those who consumed little of each kind of food little were seen in the low BMD group. It was concluded that there was a relationship between BMD and dietary habits related to calcium intake.

Key words : bone mineral density, diet, middle-aged and elderly women

\section{Introduction}

In Japan, the population of bedridden elderly people is increasing as the proportion of elderly people in the population increases as a whole (Health and Welfare Statistics Association, 1997). Bone fractures are one of the major contributory factors to people becom-

\footnotetext{
${ }^{\prime} T$ sukuba Health Center, Ibaraki Prefecture

${ }^{2}$ Graduate School of Medicine, University of Tsukuba

${ }^{3}$ College of Medical Technology and Nursing, University of Tsukuba

${ }^{4}$ Institute of Community Medicine, University of Tsukuba
} 
ing bedridden. Most bone fractures in middle-aged and elderly women occur as a result of osteoporosis (Melton et al., 1997). It has been shown that quality of life deteriorates after osteoporotic bone fractures (Cook et al., 1993). As the number of elderly people with osteoporosis is expected to reach 10 million in Japan (Suzuki et al., 1998), there has been considerable scientific interest shown in the prevention of osteoporosis. Lifestyle, including dietary and exercise habits, is considered to influence the status of osteoporosis in the elderly, with abundant calcium intake known to increase bone mineral density (BMD) (Murphy et al., 1994; Soroko et al., 1994; Nordin, 1966; Hu et al., 1993; Matkocic et al., 1979). The American and European populations derive $50 \%$ or more of their calcium intake mainly from milk and daily products. Japanese people obtain calcium not only from milk and daily products, but also from other sources including green vegetables, beans, meat, fish, shellfish and seaweed (Countermeasure Office Against Habitual Diseases, Regional Health and Health Promotion Department, Health Bureau, Ministry of Health and Welfare, 1999b). Since osteoporosis develops frequently in postmenopausal women, making them more susceptible to bone fractures following a fall or accident, further investigation of ways in which to prevent osteoporosis in these older women is important. The objective of the present study was to clarify the relationship between previous dietary habits of middle-aged and elderly women and BMD.

\section{Methods}

A survey was conducted of the dietary habits of 713 women aged 50 years and over who underwent a checkup for osteoporosis at the Omiya and Kasama Public Health Centers in Ibaraki Prefecture between September 1998 and the end of March 1999. Items surveyed were frequency of intake of the following foods when the women were in their 20's, 30's and 40's: milk, cheese, yogurt, small fish (e.g., sand lance, shishamo, baby sardines), eggs, cooked beans, tofu (bean curd), natto (fermented soybeans), green vegetables (e. g., komatsuna, shun-giku, and leafy vegetables such as spinach) and seaweed (e. g., wakame, hijiki, and kelp). BMD determined from $\Sigma \mathrm{GS} / \mathrm{D}$ values measured by the digital image-processing (DIP) method (Matsumoto et al., 1994) using a simple X-ray image of the second metacarpal of the right hand. Those women with $\Sigma \mathrm{GS} / \mathrm{D}$ values $<2.3$, which implies a high risk of development of osteoporosis, were designated as the low BMD group, and those with $\Sigma$ GS/D values $\geq 2.3$ were designated as the control group. The differences in the intake frequencies of the aforementioned foods by the two groups were evaluated by the chi-square tests. The analyses were performed using the statistical analysis software, SAS (Users Guide, 1990).

\section{Results}

Table 1 shows the proportion of women in each group that consumed the aforementioned food products in their 20's, 30's and 40 's. Significantly more women in the control group than in the low BMD group drank milk every day when they were in their 20's, 30 's and 40's ( $\mathrm{p}<0.01)$, and more women in the low BMD group had seldom drunk milk. The proportion of subjects that ate cheese 1 2 times/week when they were in 30's and 
Table 1 Frequency of intake of various foods in the past and current BMD.

1. Milk

\begin{tabular}{|c|c|c|c|c|c|}
\hline $\begin{array}{l}\text { Age group } \\
\text { (years) }\end{array}$ & Response & $\begin{array}{l}\text { Low BMD group } \\
(\Sigma \mathrm{GS} / \mathrm{D}<2.3)\end{array}$ & $\begin{array}{l}\text { Control group } \\
(\Sigma \mathrm{GS} / \mathrm{D} \geqq 2.3)\end{array}$ & \multicolumn{2}{|c|}{ Statistical test } \\
\hline \multirow[t]{4}{*}{$\cdot 20^{\prime} \mathrm{s}$} & a) drank everyday & $35(14.4 \%)$ & $84(19.1 \%)$ & \multirow{4}{*}{$\begin{array}{c}\chi^{2}=10.634 \\
\mathrm{df}=3\end{array}$} & \multirow[t]{4}{*}{$\mathrm{p}=0.014$} \\
\hline & b) drank 3 or more times/week & $22(9.1 \%)$ & $49(11.1 \%)$ & & \\
\hline & c) drank 1-2 times/week & $33(13.6 \%)$ & $86(19.6 \%)$ & & \\
\hline & d) seldom drank & $153(63.0 \%)$ & $220(50.0 \%)$ & & \\
\hline \multirow[t]{4}{*}{$\cdot 30^{\circ} \mathrm{s}$} & a) drank everyday & $42(17.3 \%)$ & $97(22.0 \%)$ & \multirow{4}{*}{$\begin{array}{c}\chi^{2}=18.777 \\
\mathrm{df}=3\end{array}$} & \multirow[t]{4}{*}{$\mathrm{p}=0.001$} \\
\hline & b) drank 3 or more times/week & $30(12.4 \%)$ & $64(14.5 \%)$ & & \\
\hline & c) drank 1-2 times/week & $36(14.8 \%)$ & $108(24.5 \%)$ & & \\
\hline & d) seldom drank & $135(55.6 \%)$ & $172(39.0 \%)$ & & \\
\hline \multirow[t]{4}{*}{$\cdot 40$ 's } & a) drank everyday & $77(31.4 \%)$ & $168(37.8 \%)$ & \multirow{4}{*}{$\begin{array}{c}\chi^{2}=17.640 \\
\mathrm{df}=3\end{array}$} & \multirow[t]{4}{*}{$\mathrm{p}=0.001$} \\
\hline & b) drank 3 or more times/week & $33(13.5 \%)$ & $84(18.9 \%)$ & & \\
\hline & c) drank 1-2 times/week & $43(17.6 \%)$ & $91(20.5 \%)$ & & \\
\hline & d) seldom drank & $92(37.6 \%)$ & $101(22.8 \%)$ & & \\
\hline
\end{tabular}

\section{Cheese}

\begin{tabular}{|c|c|c|c|c|c|}
\hline $\begin{array}{l}\text { Age group } \\
\text { (years) }\end{array}$ & Response & $\begin{array}{l}\text { Low BMD group } \\
(\Sigma \mathrm{GS} / \mathrm{D}<2.3)\end{array}$ & $\begin{array}{l}\text { Control group } \\
(\Sigma G S / D \geqq 2.3)\end{array}$ & \multicolumn{2}{|c|}{ Statistical test } \\
\hline \multirow[t]{4}{*}{$\cdot 20$ 's } & a) ate everyday & $2(0.8 \%)$ & $2(0.5 \%)$ & \multirow{4}{*}{$\begin{array}{r}\chi^{2}=1.067 \\
\mathrm{df}=3\end{array}$} & \multirow[t]{4}{*}{$\mathrm{p}=0.785$} \\
\hline & b) ate 3 or more times/week & $6(2.5 \%)$ & $16(3.7 \%)$ & & \\
\hline & c) ate 1-2 times/week & $48(19.7 \%)$ & $84(19.2 \%)$ & & \\
\hline & d) seldom ate & $188(77.1 \%)$ & $336(76.7 \%)$ & & \\
\hline \multirow[t]{4}{*}{$\cdot 30^{\prime} \mathrm{s}$} & a) ate everyday & $4(1.7 \%)$ & $3(0.7 \%)$ & \multirow{4}{*}{$\begin{array}{c}\chi^{2}=9.534 \\
d f=3\end{array}$} & \multirow[t]{4}{*}{$\mathrm{p}=0.023$} \\
\hline & b) ate 3 or more times/week & $9(3.7 \%)$ & $15(3.4 \%)$ & & \\
\hline & c) ate 1-2 times/week & $49(20.2 \%)$ & $133(30.5 \%)$ & & \\
\hline & d) seldom ate & $181(74.5 \%)$ & $285(65.4 \%)$ & & \\
\hline \multirow[t]{4}{*}{$\cdot 40^{\prime} \mathrm{s}$} & a) ate everyday & $8(3.3 \%)$ & $13(3.0 \%)$ & \multirow{4}{*}{$\begin{array}{r}\chi^{2}=9.308 \\
d f=3\end{array}$} & \multirow[t]{4}{*}{$p=0.025$} \\
\hline & b) ate 3 or more times/week & $19(7.9 \%)$ & $37(8.4 \%)$ & & \\
\hline & c) ate 1-2 times/week & $70(29.1 \%)$ & $177(40.2 \%)$ & & \\
\hline & d) seldom ate & $144(59.8 \%)$ & $213(48.4 \%)$ & & \\
\hline \multicolumn{6}{|l|}{ 3. Yogurt } \\
\hline $\begin{array}{l}\text { Age group } \\
\text { (years) }\end{array}$ & Response & $\begin{array}{l}\text { Low BMD group } \\
(\Sigma \mathrm{GS} / \mathrm{D}<2.3)\end{array}$ & $\begin{array}{l}\text { Control group } \\
(\Sigma \mathrm{GS} / \mathrm{D} \geqq 2.3)\end{array}$ & \multicolumn{2}{|c|}{ Statistical test } \\
\hline \multirow[t]{4}{*}{$\cdot 20^{\prime} \mathrm{s}$} & a) ate everyday & $15(6.1 \%)$ & $10(2.3 \%)$ & \multirow{4}{*}{$\begin{array}{c}\chi^{2}=7.993 \\
\mathrm{df}=3\end{array}$} & \multirow[t]{4}{*}{$\mathrm{p}=0.046$} \\
\hline & b) ate 3 or more times/week & $15(6.1 \%)$ & $36(8.2 \%)$ & & \\
\hline & c) ate 1-2 times/week & $40(16.2 \%)$ & $84(19.1 \%)$ & & \\
\hline & d) seldom ate & $177(71.7 \%)$ & $310(70.5 \%)$ & & \\
\hline \multirow[t]{4}{*}{$\cdot 30^{\prime} \mathrm{s}$} & a) ate everyday & $12(4.9 \%)$ & $15(3.4 \%)$ & \multirow{4}{*}{$\begin{array}{c}\chi^{2}=5.342 \\
\mathrm{df}=3\end{array}$} & \multirow[t]{4}{*}{$\mathrm{p}=0.148$} \\
\hline & b) ate 3 or more times/week & $20(8.1 \%)$ & $55(12.6 \%)$ & & \\
\hline & c) ate 1-2 times/week & $52(21.1 \%)$ & $106(24.2 \%)$ & & \\
\hline & d) seldom ate & $163(66.0 \%)$ & $262(59.8 \%)$ & & \\
\hline \multirow[t]{4}{*}{$\cdot 40^{\prime} \mathrm{s}$} & a) ate everyday & $20(8.1 \%)$ & $63(14.3 \%)$ & \multirow{4}{*}{$\begin{array}{c}\chi^{2}=16.524 \\
\mathrm{df}=3\end{array}$} & \multirow[t]{4}{*}{$\mathrm{p}=0.001$} \\
\hline & b) ate 3 or more times/week & $41(16.5 \%)$ & $87(19.7 \%)$ & & \\
\hline & c) ate 1-2 times/week & $74(29.8 \%)$ & $155(35.1 \%)$ & & \\
\hline & d) seldom ate & $113(45.6 \%)$ & $137(31.0 \%)$ & & \\
\hline
\end{tabular}


4. Small fish (e.g., sand lance, shishamo, and baby sardines)

\begin{tabular}{|c|c|c|c|c|c|}
\hline $\begin{array}{l}\text { Age group } \\
\text { (years) }\end{array}$ & Response & $\begin{array}{l}\text { Low BMD group } \\
(\Sigma \mathrm{GS} / \mathrm{D}<2.3)\end{array}$ & $\begin{array}{l}\text { Control group } \\
(\Sigma \mathrm{GS} / \mathrm{D} \geqq 2.3)\end{array}$ & \multicolumn{2}{|c|}{ Statistical test } \\
\hline \multirow[t]{4}{*}{$\cdot 20^{\prime} \mathrm{s}$} & a) ate everyday & $18(7.4 \%)$ & $46(10.4 \%)$ & \multirow{4}{*}{$\begin{array}{c}\chi^{2}=3.932 \\
\mathrm{df}=3\end{array}$} & \multirow[t]{4}{*}{$\mathrm{p}=0.269$} \\
\hline & b) ate 3 or more times/week & $45(18.4 \%)$ & $91(20.6 \%)$ & & \\
\hline & c) ate $1-2$ times/week & $112(45.7 \%)$ & $201(45.5 \%)$ & & \\
\hline & d) seldom ate & $70(28.6 \%)$ & $103(23.3 \%)$ & & \\
\hline \multirow[t]{4}{*}{$\cdot 30^{\prime} \mathrm{s}$} & a) ate everyday & $19(7.8 \%)$ & $47(10.6 \%)$ & \multirow{4}{*}{$\begin{array}{c}\chi^{2}=12.638 \\
\quad \mathrm{df}=3\end{array}$} & \multirow[t]{4}{*}{$\mathrm{p}=0.005$} \\
\hline & b) ate 3 or more times/week & $50(20.4 \%)$ & $122(27.6 \%)$ & & \\
\hline & c) ate 1-2 times/week & $123(50.2 \%)$ & $217(49.1 \%)$ & & \\
\hline & d) seldom ate & $53(21.6 \%)$ & $56(12.7 \%)$ & & \\
\hline \multirow[t]{4}{*}{$\cdot 40$ 's } & a) ate everyday & $39(16.2 \%)$ & $76(17.1 \%)$ & \multirow{4}{*}{$\begin{array}{c}\chi^{2}=10.229 \\
\mathrm{df}=3\end{array}$} & \multirow[t]{4}{*}{$\mathrm{p}=0.017$} \\
\hline & b) ate 3 or more times/week & $69(28.6 \%)$ & $158(35.5 \%)$ & & \\
\hline & c) ate 1-2 times/week & $106(44.0 \%)$ & $188(42.3 \%)$ & & \\
\hline & d) seldom ate & $27(11.2 \%)$ & $23(5.2 \%)$ & & \\
\hline \multicolumn{6}{|c|}{ 5. Meat, fish, and eggs } \\
\hline $\begin{array}{l}\text { Age group } \\
\text { (years) }\end{array}$ & Response & $\begin{array}{c}\text { Low BMD group } \\
\left(\sum \mathrm{GS} / \mathrm{D}<2.3\right)\end{array}$ & $\begin{array}{l}\text { Control group } \\
(\Sigma \mathrm{GS} / \mathrm{D} \geqq 2.3)\end{array}$ & \multicolumn{2}{|c|}{ Statistical test } \\
\hline \multirow[t]{4}{*}{$\cdot 20^{\prime} \mathrm{s}$} & a) ate everyday & $74(30.0 \%)$ & $183(41.2 \%)$ & \multirow{4}{*}{$\begin{array}{c}\chi^{2}=11.062 \\
d f=3\end{array}$} & \multirow{4}{*}{$\mathrm{p}=0.011$} \\
\hline & b) ate 3 or more times/week & $83(33.6 \%)$ & $133(30.0 \%)$ & & \\
\hline & c) ate 1-2 times/week & $70(28.3 \%)$ & $109(24.6 \%)$ & & \\
\hline & d) seldom ate & $20(8.1 \%)$ & $19(4.3 \%)$ & & \\
\hline \multirow[t]{4}{*}{$\cdot 30^{\prime} \mathrm{s}$} & a) ate everyday & $81(33.1 \%)$ & $211(48.0 \%)$ & \multirow{4}{*}{$\begin{array}{c}\chi^{2}=29.412 \\
\mathrm{df}=3\end{array}$} & \multirow{4}{*}{$\mathrm{p}=0.001$} \\
\hline & b) ate 3 or more times/week & $84(34.3 \%)$ & $140(31.8 \%)$ & & \\
\hline & c) ate $1-2$ times/week & $62(25.3 \%)$ & $84(19.1 \%)$ & & \\
\hline & d) seldom ate & $18(7.4 \%)$ & $5(1.1 \%)$ & & \\
\hline \multirow[t]{4}{*}{$\cdot 40^{\prime} \mathrm{s}$} & a) ate everyday & $96(39.3 \%)$ & $254(57.1 \%)$ & \multirow{4}{*}{$\begin{array}{c}\chi^{2}=31.614 \\
\mathrm{df}=3\end{array}$} & \multirow[t]{4}{*}{$\mathrm{p}=0.001$} \\
\hline & b) ate 3 or more times/week & $86(35.3 \%)$ & $135(30.3 \%)$ & & \\
\hline & c) ate $1-2$ times/week & $52(21.3 \%)$ & $54(12.1 \%)$ & & \\
\hline & d) seldom ate & $10(4.1 \%)$ & $2(0.5 \%)$ & & \\
\hline \multicolumn{6}{|c|}{ 6. Cooked beans } \\
\hline $\begin{array}{l}\text { Age group } \\
\text { (years) }\end{array}$ & Response & $\begin{array}{c}\text { Low BMD group } \\
(\Sigma \mathrm{GS} / \mathrm{D}<2.3)\end{array}$ & $\begin{array}{l}\text { Control group } \\
(\Sigma G S / D \geqq 2.3)\end{array}$ & \multicolumn{2}{|c|}{ Statistical test } \\
\hline \multirow[t]{4}{*}{$\cdot 20^{\prime} \mathrm{s}$} & a) ate everyday & $8(3.4 \%)$ & $10(2.3 \%)$ & \multirow{4}{*}{$\begin{array}{c}\chi^{2}=2.929 \\
d f=3\end{array}$} & \multirow[t]{4}{*}{$\mathrm{p}=0.403$} \\
\hline & b) ate 3 or more times/week & $10(4.2 \%)$ & $31(7.2 \%)$ & & \\
\hline & c) ate $1-2$ times/week & $132(55.5 \%)$ & $237(54.7 \%)$ & & \\
\hline & d) seldom ate & $88(37.0 \%)$ & $154(35.6 \%)$ & & \\
\hline$\cdot 30^{\prime} \mathrm{s}$ & a) ate everyday & $6(2.5 \%)$ & $10(2.3 \%)$ & $\chi^{2}=1.330$ & $\mathrm{p}=0.722$ \\
\hline & b) ate 3 or more times/week & $17(7.1 \%)$ & $40(9.3 \%)$ & $\mathrm{df}=3$ & \\
\hline & c) ate 1-2 times/week & $145(60.9 \%)$ & $264(61.5 \%)$ & & \\
\hline & d) seldom ate & $70(29.4 \%)$ & $114(26.6 \%)$ & & \\
\hline$\cdot 40^{\prime} \mathrm{s}$ & a) ate everyday & $8(3.4 \%)$ & $17(3.9 \%)$ & $\chi^{2}=9.440$ & $\mathrm{p}=0.024$ \\
\hline & b) ate 3 or more times/week & $19(8.0 \%)$ & $60(13.9 \%)$ & $\mathrm{df}=3$ & \\
\hline & c) ate 1-2 times/week & $152(64.1 \%)$ & $283(65.4 \%)$ & & \\
\hline & d) seldom ate & $58(24.5 \%)$ & $72(16.6 \%)$ & & \\
\hline
\end{tabular}


7. Tofu(bean curd)

\begin{tabular}{|c|c|c|c|c|c|}
\hline $\begin{array}{l}\text { Age group } \\
\text { (years) }\end{array}$ & Response & $\begin{array}{l}\text { Low BMD group } \\
(\Sigma \mathrm{GS} / \mathrm{D}<2.3)\end{array}$ & $\begin{array}{l}\text { Control group } \\
(\Sigma \mathrm{GS} / \mathrm{D} \geqq 2.3)\end{array}$ & \multicolumn{2}{|c|}{ Statistical test } \\
\hline \multirow[t]{4}{*}{$\cdot 20^{\prime} \mathrm{s}$} & a) ate everyday & $26(10.4 \%)$ & $29(6.6 \%)$ & \multirow{4}{*}{$\begin{array}{r}\chi^{2}=9.626 \\
\mathrm{df}=3\end{array}$} & \multirow[t]{4}{*}{$p=0.022$} \\
\hline & b) ate 3 or more times/week & $65(26.1 \%)$ & $131(29.7 \%)$ & & \\
\hline & c) ate 1-2 times/week & $128(51.4 \%)$ & $251(57.0 \%)$ & & \\
\hline & d) seldom ate & $30(12.1 \%)$ & $30(6.8 \%)$ & & \\
\hline \multirow[t]{4}{*}{$\cdot 30^{\prime} \mathrm{s}$} & a) ate everyday & $26(10.4 \%)$ & $32(7.3 \%)$ & \multirow{4}{*}{$\begin{array}{c}\chi^{2}=12.569 \\
d f=3\end{array}$} & \multirow[t]{4}{*}{$\mathrm{p}=0.006$} \\
\hline & b) ate 3 or more times/week & $70(26.1 \%)$ & $167(38.1 \%)$ & & \\
\hline & c) ate 1-2 times/week & $135(51.4 \%)$ & $223(50.9 \%)$ & & \\
\hline & d) seldom ate & $20(12.1 \%)$ & $16(3.7 \%)$ & & \\
\hline \multirow{4}{*}{$\cdot 40$ 's } & a) ate everyday & $36(14.3 \%)$ & $64(14.5 \%)$ & \multirow{4}{*}{$\begin{array}{c}\chi^{2}=19.024 \\
\mathrm{df}=3\end{array}$} & \multirow[t]{4}{*}{$p=0.001$} \\
\hline & b) ate 3 or more times/week & $86(34.1 \%)$ & $204(46.1 \%)$ & & \\
\hline & c) ate $1-2$ times/week & $119(47.2 \%)$ & $172(38.8 \%)$ & & \\
\hline & d) seldom ate & $11(4.4 \%)$ & $3(0.7 \%)$ & & \\
\hline \multicolumn{6}{|c|}{ 8. Natto (fermented soybeans) } \\
\hline $\begin{array}{l}\text { Age group } \\
\text { (years) }\end{array}$ & Response & $\begin{array}{l}\text { Low BMD group } \\
(\Sigma \mathrm{GS} / \mathrm{D}<2.3)\end{array}$ & $\begin{array}{l}\text { Control group } \\
(\Sigma G S / D \geqq 2.3)\end{array}$ & \multicolumn{2}{|c|}{ Statistical test } \\
\hline \multirow{4}{*}{$\cdot 20^{\prime} \mathrm{s}$} & a) ate everyday & $23(9.3 \%)$ & $31(7.0 \%)$ & \multirow{4}{*}{$\begin{array}{c}\chi^{2}=8.059 \\
\mathrm{df}=3\end{array}$} & \multirow{4}{*}{$p=0.045$} \\
\hline & b) ate 3 or more times/week & $56(22.7 \%)$ & $112(25.4 \%)$ & & \\
\hline & c) ate $1-2$ times/week & $112(45.3 \%)$ & $231(52.4 \%)$ & & \\
\hline & d) seldom ate & $56(22.7 \%)$ & $67(15.2 \%)$ & & \\
\hline \multirow[t]{4}{*}{$\cdot 30^{\prime} \mathrm{s}$} & a) ate everyday & $25(10.0 \%)$ & $32(7.3 \%)$ & \multirow{4}{*}{$\begin{array}{r}\chi^{2}=7.972 \\
\mathrm{df}=3\end{array}$} & \multirow{4}{*}{$p=0.047$} \\
\hline & b) ate 3 or more times/week & $65(26.1 \%)$ & $145(32.9 \%)$ & & \\
\hline & c) ate 1-2 times/week & $124(49.8 \%)$ & $225(51.0 \%)$ & & \\
\hline & d) seldom ate & $35(14.1 \%)$ & $39(8.8 \%)$ & & \\
\hline \multirow[t]{4}{*}{$\cdot 40^{\prime} \mathrm{s}$} & a) ate everyday & $50(20.0 \%)$ & $72(16.2 \%)$ & \multirow{4}{*}{$\begin{array}{c}\chi^{2}=17.038 \\
\mathrm{df}=3\end{array}$} & \multirow[t]{4}{*}{$p=0.001$} \\
\hline & b) ate 3 or more times/week & $71(28.4 \%)$ & $189(42.6 \%)$ & & \\
\hline & c) ate $1-2$ times/week & $113(45.2 \%)$ & $171(38.5 \%)$ & & \\
\hline & d) seldom ate & $16(6.4 \%)$ & $12(2.7 \%)$ & & \\
\hline \multicolumn{6}{|c|}{ 9. Green Vegetables (e.g., komatsuna, shun-giku, and leafy vegetables such as spinach) } \\
\hline $\begin{array}{l}\text { Age group } \\
\text { (years) }\end{array}$ & Response & $\begin{array}{l}\text { Low BMD group } \\
(\Sigma \mathrm{GS} / \mathrm{D}<2.3)\end{array}$ & $\begin{array}{l}\text { Control group } \\
(\Sigma \mathrm{GS} / \mathrm{D} \geqq 2.3)\end{array}$ & \multicolumn{2}{|c|}{ Statistical test } \\
\hline \multirow[t]{4}{*}{$\cdot 20^{\prime} \mathrm{s}$} & a) ate everyday & $151(59.9 \%)$ & $256(57.5 \%)$ & \multirow{4}{*}{\multicolumn{2}{|c|}{$\begin{array}{c}\chi^{2}=9.440 \quad p=0.024 \\
d f=3\end{array}$}} \\
\hline & b) ate 3 or more times/week & $56(22.2 \%)$ & $113(25.4 \%)$ & & \\
\hline & c) ate $1-2$ times/week & $37(14.7 \%)$ & $69(15.5 \%)$ & & \\
\hline & d) seldom ate & $7(2.8 \%)$ & $7(1.6 \%)$ & & \\
\hline \multirow[t]{4}{*}{$\cdot 30^{\prime} \mathrm{s}$} & a) ate everyday & $167(66.3 \%)$ & $269(61.1 \%)$ & $\chi^{2}=6.333$ & $\mathrm{p}=0.093$ \\
\hline & b) ate 3 or more times/week & $52(20.6 \%)$ & $116(26.4 \%)$ & $\mathrm{df}=3$ & \\
\hline & c) ate $1-2$ times/week & $31(12.3 \%)$ & $55(12.5 \%)$ & & \\
\hline & d) seldom ate & $2(0.8 \%)$ & $0(0.0 \%)$ & & \\
\hline$\cdot 40^{\prime} \mathrm{s}$ & a) ate everyday & $172(68.3 \%)$ & $304(68.5 \%)$ & $\chi^{2}=5.722$ & $\mathrm{p}=0.057$ \\
\hline & b) ate 3 or more times/week & $47(18.7 \%)$ & $104(23.4 \%)$ & $\mathrm{df}=3$ & \\
\hline & c) ate 1-2 times/week & $33(13.1 \%)$ & $36(8.1 \%)$ & & \\
\hline & d) seldom ate & $0(0.0 \%)$ & $0(0.0 \%)$ & & \\
\hline
\end{tabular}


10. Seaweed (e.g., wakame, hijiki, and kelp)

\begin{tabular}{|c|c|c|c|c|c|}
\hline $\begin{array}{l}\text { Age group } \\
\text { (years) }\end{array}$ & Response & $\begin{array}{l}\text { Low BMD group } \\
(\Sigma \mathrm{GS} / \mathrm{D}<2.3)\end{array}$ & $\begin{array}{l}\text { Control group } \\
(\Sigma G S / D \geqq 2.3)\end{array}$ & \multicolumn{2}{|c|}{ Statistical test } \\
\hline \multirow[t]{4}{*}{$\cdot 20$ 's } & a) ate everyday & $25(10.1 \%)$ & $48(10.8 \%)$ & \multirow{4}{*}{$\begin{array}{c}\chi^{2}=5.635 \\
\mathrm{df}=3\end{array}$} & \multirow[t]{4}{*}{$\mathrm{p}=0.131$} \\
\hline & b) ate 3 or more times/week & $76(30.8 \%)$ & $151(33.9 \%)$ & & \\
\hline & c) ate $1-2$ times/week & $117(47.4 \%)$ & $218(49.0 \%)$ & & \\
\hline & d) seldom ate & $28(11.3 \%)$ & $28(6.3 \%)$ & & \\
\hline \multirow[t]{4}{*}{$\cdot 30$ 's } & a) ate everyday & $27(10.9 \%)$ & $51(11.5 \%)$ & \multirow{4}{*}{$\begin{array}{c}\chi^{2}=15.422 \\
\mathrm{df}=3\end{array}$} & \multirow[t]{4}{*}{$\mathrm{p}=0.00$} \\
\hline & b) ate 3 or more times/week & $82(33.1 \%)$ & $188(42.5 \%)$ & & \\
\hline & c) ate 1-2 times/week & $119(48.0 \%)$ & $192(43.4 \%)$ & & \\
\hline & d) seldom ate & $20(8.1 \%)$ & $11(2.5 \%)$ & & \\
\hline \multirow[t]{4}{*}{$\cdot 40^{\prime} \mathrm{s}$} & a) ate everyday & $38(15.4 \%)$ & $92(20.6 \%)$ & \multirow{4}{*}{$\begin{array}{c}\chi^{2}=22.158 \\
\mathrm{df}=3\end{array}$} & \multirow[t]{4}{*}{$\mathrm{p}=0.001$} \\
\hline & b) ate 3 or more times/week & $99(40.1 \%)$ & $210(47.1 \%)$ & & \\
\hline & c) ate $1-2$ times/week & $102(41.3 \%)$ & $144(32.3 \%)$ & & \\
\hline & d) seldom ate & $8(3.2 \%)$ & $0(0.0 \%)$ & & \\
\hline
\end{tabular}

40's was higher in the control group $(\mathrm{p}<0.05)$, and more women in the low BMD group had seldom eaten cheese. A greater proportion of subjects in the control group had eaten yogurt 1-2 times/week when they were in their 20 's, 30's and 40's ( $p<0.05)$, and there were more women in the low BMD group who had seldom eaten yogurt in their 30's and 40's. The proportion of subjects that ate small fish every day or three or more times per week when they were in their 30's and 40's was higher in the control group $(\mathrm{p}<0.05)$, with more women who had rarely eaten fish in the low BMD group. Significantly more women in the control group ate meat, fish and eggs everyday in their 20's, 30's and 40's $(p<0.05)$, with a greater number of subjects in the low BMD group having seldom eaten these products. There were slightly more subjects in the control group that often ate cooked beans in their 40's $(\mathrm{p}<0.05)$. Tofu consumption three or more times per week by subjects in their 20's, 30's and 40's was slightly higher in the control group $(\mathrm{p}<0.05)$, and more women in the low BMD group had eaten virtually no tofu. The proportion of subjects who ate natto 3 or 4 times per week was higher in the control group $(\mathrm{p}<0.05)$. More women in the control group had eaten green vegetables three or more times per week when they were in their 20's $(p<0.05)$. A greater number of women in the control group consumed seaweed daily or three or more times per week in their 30's and 40's. More women in the low BMD group had seldom eaten seaweed in their 20's, 30's and 40's $(\mathrm{p}<0.01)$.

Fig. 1 shows that the proportion of women who had seldom eaten certain food products in the past was greater in the low BMD group than in the control group.

\section{Discussion}

Women aged 65 years or more tend to develop lumbago and bone fractures due to osteoporosis (Ross et al., 1988). In addition, from around the age of fifty, women tend to secrete less estrogen, which has an important effect on bone metablism (Minaguchi and Gorai, 1993). Therefore, to investigate the relationship between lifestyle and $\mathrm{BMD}$ for prevention of osteoporosis, the target group 
for this survey was limited to women aged fifty years and older.

The Public Hygiene Council published the sixth revision of the Nutritional Requirements for Japanese in October 1999. The recommended dietary requirement for calcium is $600 \mathrm{mg} /$ day for adult females, irrespective of age and activity level (Health and Nutrition Research Committee, 1999). The daily intake of calcium for Japanese increased steadily for 25 years following World War II, before leveling off at around $550 \mathrm{mg}$ in 1975. The average daily intake was determined to be $579 \mathrm{mg}$ by a survey in 1997, making calcium the only nutrient with an intake found to be below the daily requirement. The Ministry of Health and Welfare has recognized the need to promote increased calcium intake in order to reduce the development of osteoporosis (Ministry of Health and Welfare, 1996).

Several studies have reported that calcium

\section{Milk}

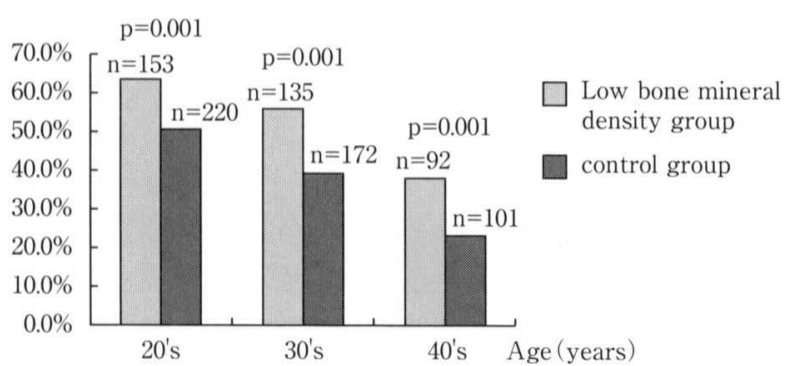

3. Yogurt

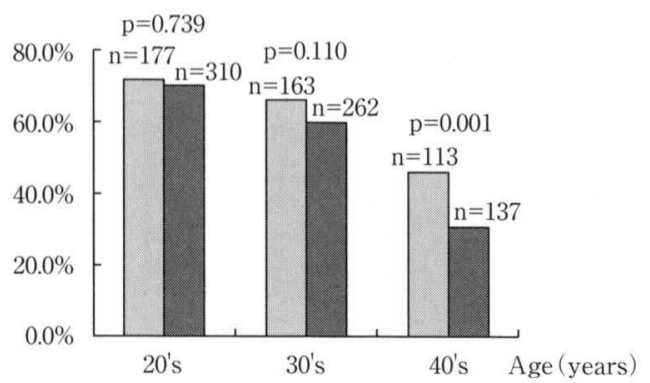

5. Meat, Fish, Egg

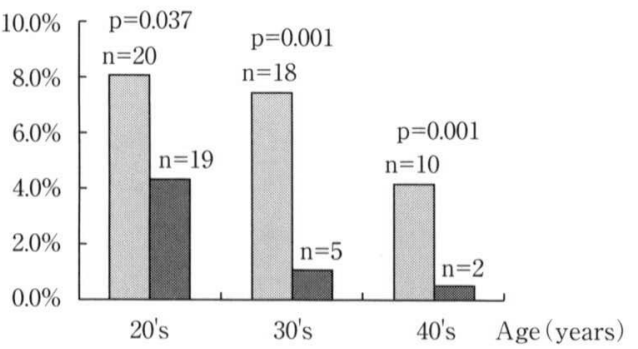

2. Cheese

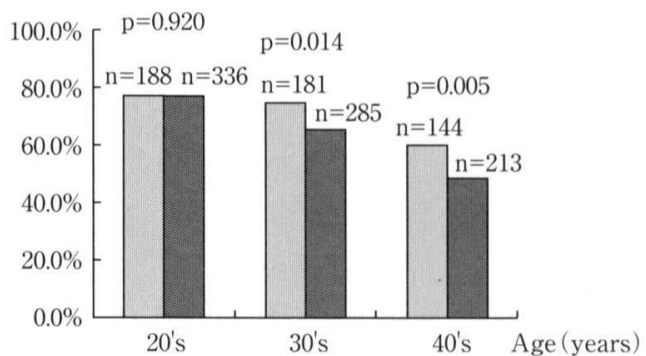

4. Small fish

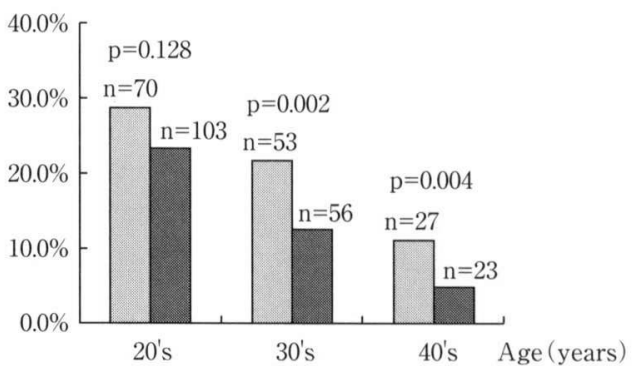

6. Cooked beans

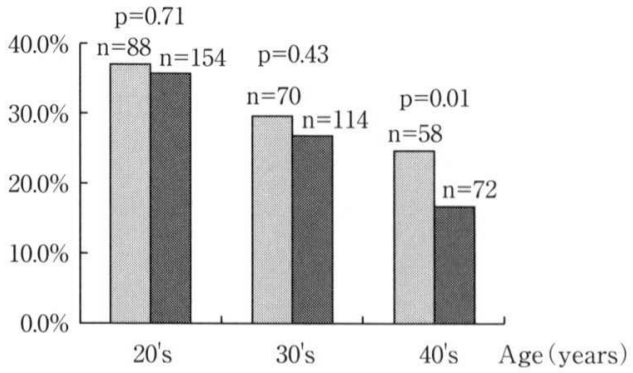




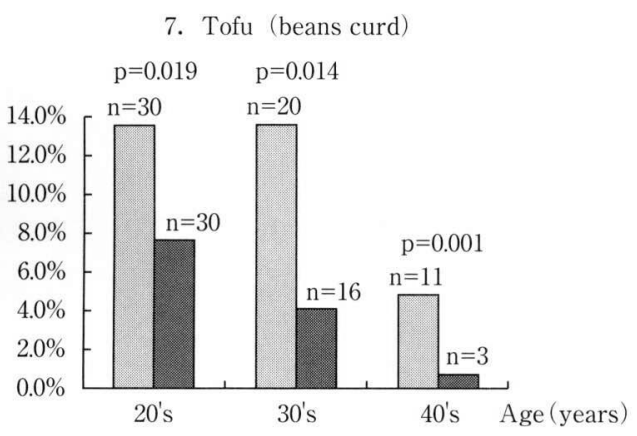

9. Green Vegetables

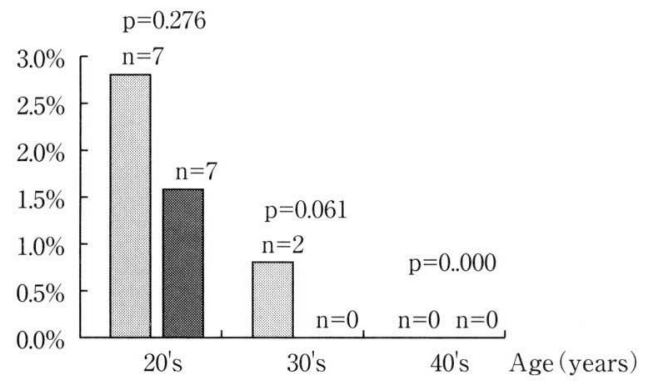

8. Natto (fermented beans)

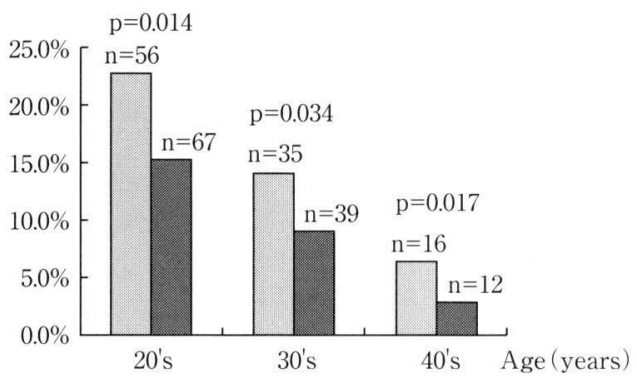

10. Seaweed

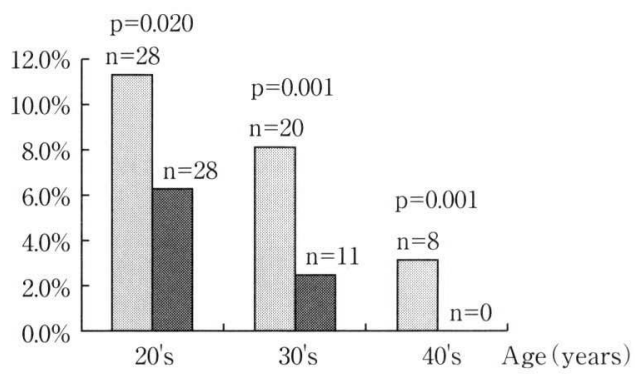

Fig. 1 The proportion of people who seldom consumed certain food in the past. The numbers correspond to the numbers of foods in Table 1. Degrees of freedom for each chi square test are one.

intake has a preventive effect on osteoporosis (Cumming, 1990; Matkovic, 1991; Nordin, 1966). A bottle of milk $(200 \mathrm{ml})$ and a piece of cheese $(30 \mathrm{~g})$ contain approximately $200 \mathrm{mg}$ of calcium. Absorption of calcium from milk and cheese is high, at approximately $50 \%$. Marine food products, such as small fish and seaweed, as well as green leafy vegetables (absorption rate $20 \%$ ) contain considerable amounts of calcium, with Kanematsu (1953) reporting that the absorption rate of calcium from small fish is $38 \%$ and that from vegetables is $18 \%$. According to a survey in $\mathrm{Yu}$ goslavia of women aged 65 years and more, those residing in areas with low average calcium and protein intake were reported to have a markedly higher incidence of bone fractures (Matkovic et al., 1979). Miyamura et al. (1994) reported that frequency of intake of milk and other dairy products is positively associated with the amount of calcium intake, which is supported by the present study's findings that a greater number of women seldom drank milk in the low BMD group compared to the controls. This result indicates that milk effectively prevents the development of osteoporosis. Ezawa et al. (1992) recommend that Japanese people who tend to have lower than average calcium intakes and who do not like dairy products should add small fish to their diet. On the other hand, they found no relationship between calcium intake and frequency of beans, green leafy vegetables, milk, dairy products, seaweed, fish and shellfish consumption. In addition, no significant relation- 
ship was found between bone mineral content and food-based calcium intake in the study conducted by Tsuchida et al. (1998). However, when total daily calcium intake was less than $600 \mathrm{mg}$, a significant correlation was found between the amount of small fish consumption and bone mineral content. In contrast, Hosokawa et al. (1996a) found in their survey that frequency of small fish and seaweed consumption was not related to incidences of osteoporosis or BMD. A correlation was observed only with consumption of milk and, accordingly, Hosokawa et al. (1996b) proposed that the rate of absorption of calcium from milk is better than that from small fish. Hirota (1997) cautioned against excessive intake of sodium, however, when he recommended intake of plenty of small fish for prevention of adult disease, because salt content in small fish is high, and sodium tends to accelerate urinary calcium excretion. It follows that consuming milk plays more important role in prevention of osteoporosis than the other foods.

The current study also revealed that women had healthier eating habits in their 40 's than in their 20's. We therefore advocate dissemination of information promoting routine intake of calcium in the diet of women from their 20's, because eating habits are extremely difficult to alter in elderly people.

In recent years, vitamin $\mathrm{K}$ has been found to play an important role in maintaining bone mass and promoting bone repair. A study was conducted in an area famous for the production of natto, which contains an abundance of vitamin $\mathrm{K}$ (The Ministry of Health and Welfare Recommended Dietary Allowance of Japanese (5th revision), 1994). This study identified a relationship between natto intake and BMD. According to previously published reports, vitamin $\mathrm{K}$ accelerated repair of bone fractures in a rabbit model. Kanai et al. (1995) reported that in a doubleblind test, the oral administration of vitamin $\mathrm{K}_{2}$ (menaquinone-4; $45 \mathrm{mg}$ /day) significantly increased bone mass in elderly women with osteoporosis. It has been confirmed that vitamin $\mathrm{K}$ induces the increase in BMD mainly on cortical bone (Knapen et al., 1989).

This and similar studies suggested that encouraging good dietary habits, particularly from a young age, will aid in preventing of osteoporosis. We also suggest that it is important to disseminate accurate information about this disease not only to elderly people but to everyone. Urging people to establish healthy dietary habits contributes not only to the prevention of osteoporosis but also to the prevention of other diseases that occur as a result of poor lifestyle.

\section{Conclusions}

Women with normal BMD had more frequently consumed food products that contained large amounts of calcium than those with low BMD. Given these findings, we suggest that a correlation exists between BMD and frequency of intake of foods rich in calcium.

\section{Acknowledgments}

The authors thank the staff of the Omiya and Kasama Public Health Centers, Ibaraki Prefecture for their assistance with the study.

\section{References}

Consensus development conference (1993): Diagnosis, prophylaxis, and treatment of osteoporo- 
sis. Am. J. Med., 94, 646

Cook DJ, Guyatt GH, Adachi JD et al. (1993): Quality of life issues in women with vertebral fractures due to osteoporosis. Arthritis. Rheum., 36(6), 750-756

Countermeasure Office Against Habitual Diseases, Regional Health and Health Promotion Department, Health Bureau, Ministry of Health and Welfare (1999a): Current Nutritional Status of People (results of nutrition survey in 1997), 3: 29, Dai-ichi Shuppan, Tokyo [in Japanese]

Countermeasure Office Against Habitual Diseases, Regional Health and Health Promotion Department, Health Bureau, Ministry of Health and Welfare (1999b): Current Nutrition Status of People (results of nutrition survey in 1997), 3: 36 Dai-ichi Shuppan, Tokyo [in Japanese]

Cumming RG (1990): Calcium intake and bone mass. A quantitative review of the evidence. Calcif. Tissue Int., 47, 194-201

Ezawa I (1992): Meals recommended for osteoporosis. Asian Med. J., 35(4), 214-217

Health and Nutrition Research Committee (1999): Required nutrition of Japanese (sixth revision). Ed. : Dai-ichi Shuppan 9 [in Japanese]

Health and Welfare Statistics Association (1997): Trends of People's Hygiene and Indices of Health and Welfare, 46(9) Kosaido Printing (Tokyo) [in Japanese].

Hiraki M (1992): Special issue on calcium metabolism and health management, relationship with osteoporosis: Health Management 1, 20-30 [in Japanese]

Hirota T (1997): Diet Therapy for Osteoporosis, Body Science 195, 56-60 [in Japanese]

Hosokawa M, Yanagi H, Kawanami K et al. (1996): Relationship between dietary lifestyle in youth and Osteoporosis: Jpn. J. Public Health, 43, 606-614 [in Japanese]

Hu JF, Zhao XH, Jia JB et al. (1993): Dietary calcium and bone density among middle-aged and elderly woman in China. Am. J. Clin. Nutr., $58,219-227$

Kanai M, Mizuno Y, Hosoi T et al. (1995): Study on serum vitamin $\mathrm{K}$ concentration and involutional osteoporosis. Jpn. J. Elderly Med., 32, 195 -200 [in Japanese]

Kanematsus (1953): Study on the calcium utiliza- tion rate in various foods in adults and calcium requirement. Nutrition and Food, 6, 135-147 [in Japanese]

Knapen MH, Hamulya'h K, Vermeer C (1989): The effects of vitamin $\mathrm{K}$ suppementation on circulating osteocalcin (Bone mineral Gla Protein) and urinary calcium excretion, Ann. Inter. Med., 111, 1001-1005

Matkovic V (1991): Calcium metabolism and calcium requirements during skeletal modeling and consolidation. Am. J. Clin. Nutr., 54 (suppl). $245 \mathrm{~S}-260 \mathrm{~S}$

Matkocic V, Kostial K, Simonovic I et al. (1979): Bone status and fracture rates in two regions of Yugoslavia. Am. J. Clin. Nutr., 32, 540-549

Matsumoto C, Kushida K, Yamazaki K et al. (1994): Metacarpal bone mass in normal and osteoporosis Japanese women using computed $\mathrm{x}$ ray densitometry. Calcif. Tissue Int., 55, 324329

Melton LJ III, Thamer M, Ray NF et al. (1997): Fractures attributable to osteoporosis: report from the National Osteoporosis Foundation, J. Bone Miner. Res., 12(1), 16-23

Minaguchi H, Gorai I (1993): Psotmenopausal osteoporosis-its definition and conception, Igaku no Ayumi, 165(9), 504-507

Ministry of health and welfare (1996): Annual result of National nutrition, Sep [in Japanese]

Miyamura H, Yamagata Z, Iijima S et al. (1994): Study on association between risk factors for osteoporosis and bone mineral density. Jpn. J. Public Health, 41, 1122-1230 [in Japanese]

Murphy S, Khaw KT, May H et al. (1994): Milk consumption and bone mineral density in middle aged and elderly women. BMJ., 308, 939941

Nordin B (1966): International patterns of osteoporosis. Clin Orthop, 45, 17-30

Ross PD, Orimo H, Washida RD et al. (1988): Methodological issues in comparing genetic and environmental influences on bone mass, Bone Miner., 7(1), 66-77

Soroko S, Holbrook TL, Edelstein S et al. (1994): Lifetime milk consumption and bone mineral density in older women. Am. J. Public Health, 84, 1319-1322

Suzuki T, Yoshida H, Ishizaki T (1998): Epidemiology of Osteoporosis: Incidence, prevalence, and prognosis. Jpn. J. Clin. Med., 56(6), 1563- 
1568

The Ministry of Health and Welfare (1994): Required Nutrition of Japanese (5th revision), The Ministry of Health and Welfare (Tokyo) [in Japanese]

Tsuchida T, Mizushima S, Takahashi H et al.
(1998): Relationship between dietary calcium and bone mineral density before menopause: $\mathrm{J}$. Public Health [in Japanese]

Users Guide (1990): Procedures, version 6 4th ed., SAS inc

(Received 4. 28, 2000 ; Accepted 3. 30, 2001) 